\title{
Mass segregation in rich LMC clusters from modelling of deep HST colour-magnitude diagrams
}

\author{
L. O. Kerber ${ }^{1,2}$ and B. X. Santiago ${ }^{1}$ \\ ${ }^{1}$ Universidade Federal do Rio Grande do Sul, IF, CP 15051, Porto Alegre 91501-970, RS, Brazil \\ e-mail: kerber@astro.iag.usp.br \\ ${ }^{2}$ Universidade de São Paulo, IAG, Rua do Matão 1226, Cidade Universitária, São Paulo 05508-900, SP, Brazil
}

Received 13 September 2005 / Accepted 10 January 2006

\begin{abstract}
Aims. We used the deep colour-magnitude diagrams (CMDs) of five rich LMC clusters (NGC 1805, NGC 1818, NGC 1831, NGC 1868, and Hodge 14) observed with HST/WFPC2 to derive their present day mass function (PDMF) and its variation with position within the cluster.

Methods. The PDMF was parameterized as a power law in the available main-sequence mass range of each cluster, typically $0.9 \lessgtr m / M_{\odot} \lesssim 2.5$; its slope was determined at different positions spanning from the very centre out to several core radii. The CMDs in the central regions of the clusters were carefully studied earlier, resulting in accurate age, metallicity, distance modulus, and reddening values. The slope $\alpha$ (where Salpeter is 2.35) was determined in annuli by following two distinct methods: 1) a power law fit to the PDMF obtained from the systemic luminosity function (LF); 2) a statistical comparison between observed and model CMDs. In the second case, $\alpha$ is a free input parameter in the CMD modelling process where we incorporate photometric errors and the effect of binarity as a fraction of unresolved binaries $\left(f_{\text {bin }}=100 \%\right)$ with random pairing of masses from the same PDMF.

Results. In all clusters, significant mass segregation is found from the positional dependence of the PDMF slope: $\alpha \lesssim 1.8$ for $R \leq 1.0 R_{\text {core }}$ and $\alpha \sim$ Salpeter inside $R=2 \sim 3 R_{\text {core }}$ (except for Hodge 14, where $\alpha \sim$ Salpeter for $R \sim 4 R_{\text {core }}$ ). The results are robust in the sense that they hold true for both methods used. The CMD method reveals that unresolved binaries flatten the PDMF obtained form the systemic LF, but this effect is smaller than the uncertainties in the $\alpha$ determination. For each cluster we estimated dynamical ages inside the core and for the entire system. In both cases we found a trend in the sense that older clusters have flatter PDMF, consistent with a dynamical mass segregation and stellar evaporation.
\end{abstract}

Key words. galaxies: star clusters - galaxies: Magellanic Clouds - stars: luminosity function, mass function stars: Hertzsprung-Russell (HR) and C-M diagrams - methods: numerical

\section{Introduction}

The phenomenon of mass segregation in a stellar system, which means a preferential concentration of high-mass stars towards the centre and a preferential allocation of lower-mass stars towards the periphery, seems to occur in systems with widely distinct physical properties. It has been detected and extensively studied in globular clusters (de Marchi \& Paresce 1996; Andreuzzi et al. 2000; Howell et al. 2000), open clusters (Raboud \& Mermilliod 1998; Durgapal \& Pandey 2001; Bonatto \& Bica 2003, 2005), and even in star forming regions (Hillenbrand \& Hartmann 1998; Stolte et al. 2002).

A key role in the investigation of mass segregation was played by the Hubble Space Telescope (HST), which for the first time resolved the stars in the very centre of the rich star clusters in the Magellanic Clouds (MCs). This provided a new "laboratory" for obtaining constraints on the physical processes involved in star formation, where the possible universality of the initial mass function (IMF) is a central issue (Kroupa 2002). The main reason is that, unlike the Galaxy, the MCs present a great variety of clusters, including young and rich star clusters.

There are two possible and distinct origins for this effect of mass segregation: dynamical and primordial. The first one is caused by the dynamical evolution of the cluster, where the stars tend to reach the equipartition of kinetic energy due to stellar encounters (Spitzer 1987; Binney \& Tremaine 1987). Therefore, the high-mass stars decrease their velocities, sinking towards the cluster centre, while the low-mass stars speed up and take higher orbits on average. In a simplified discussion, the characteristic time-scale of dynamical mass segregation in a stellar system is given by $m_{\text {low }} / m_{\text {high }}$ times the two-body relaxation time $\left(t_{\mathrm{rl}}\right)$ (Spitzer 1987), where $m_{\text {low }}$ and $m_{\text {high }}$ are the lowest and highest masses in the cluster, respectively. This relation indicates that the time when mass segregation occurs scales with $t_{\mathrm{rl}}$ and can be very short if $m_{\text {high }} \gg m_{\text {low }}$. An example of a very young ( $\$ 2 \mathrm{Myr}$ ) system that presents mass segregation, which can be interpreted as a dynamical effect, is the Orion Nebulae Cluster (ONC) (Kroupa et al. 2001).

It is also important to note that dynamical mass segregation combined with stellar evaporation may lead to the preferential loss of lower-mass stars. Since these stars are more likely populating the outermost regions of a cluster, they are more prone to be unbound due to their lower binding energy. Therefore one could expect that stellar clusters would have flatter global PDMF as they become dynamically older. In fact this effect is observed in open clusters (Bonatto \& Bica 2005) and Galactic globular clusters (Baumgardt \& Makino 2003), which are also modelled with $N$-body simulations.

On the other hand, primordial mass segregation may be a natural outcome of star-formation theory, since a protocluster with higher central density should have a greater probability of 
forming proportionally more high-mass stars in its centre. Some scenarios propose mass segregation at the onset of star formation through interactions among the protostars, since the collision probabilities increase with density (Bonnel \& Davies 1998); alternatively, accretion rates are enhanced with the mass of the accreting protostar (Behrend \& Meader 2001).

Regardless of the physical mechanism used to account for the origin of mass segregation, there are several techniques for diagnosing and quantifying the effect, the main ones being based on stellar statistics. By counting stars in different annuli, one may search for variations in radial profiles as a function of stellar-mass range or for changes in the slope of the luminosity function (LF) or of the present day mass function (PDMF). Although there are uncertainties in the conversion of stellar luminosity into mass (de Grijs et al. 2002a), the last option provides a more direct constraint on $\mathrm{N}$-body simulations that intend to recover the initial conditions of the cluster (Kroupa et al. 2001; Baumgardt \& Makino 2003; Moraux et al. 2004). Since the mass-luminosity relation is dependent on metallicity, its uncertainty may be efficiently reduced by precisely determining the cluster physical parameters, by means of careful modelling of its colour-magnitude diagram (CMD). A detailed enough CMD modelling should result in physical parameters predominantly limited by uncertainties associated to the models of stellar evolution, rather than to the data.

The main goal of this work is to determine the spatial dependence of the PDMF slope of five rich Large Magellanic Cloud (LMC) clusters, namely NGC 1805, NGC 1818, NGC 1831, NGC 1868, and Hodge 14. In Kerber \& Santiago (2005), we presented the analysis of deep CMDs from these clusters obtained with HST/WFPC2 in the $F 555 W(\sim V)$ and $F 814 W(\sim I)$ filters. Efficient use of the data was made by means of direct comparisons of the observed CMD (statistically corrected for incompleteness and field star contamination) to model ones. By modelling the CMDs in the central region of each cluster we inferred the metallicity $(Z)$, the intrinsic distance modulus $\left((m-M)_{0}\right)$ and the reddening value $(E(B-V))$. We also determined the age $(\tau)$ for NGC 1831, NGC 1868, and Hodge 14.

Santiago et al. (2001) and de Grijs et al. (2002a,b), using the same data, analyse the mass segregation in these clusters by means of the spatial dependence of the LF slope. Their diagnostic was clear: all clusters present mass segregation, even the youngest ones (NGC 1805 and NGC 1818). Meanwhile, de Grijs et al. (2002a,b) reach the same result by comparing the radial profile dependence with the stellar mass range. They also derive the PDMF and its variation with position within the cluster, but only for the youngest ones. Here we present this type of analysis for the five clusters, using the cluster parameters derived by Kerber \& Santiago (2005). The PDMF slope $(\alpha)$ in different annuli was determined by following two distinct methods: 1) power law fit to the PDMF obtained from the systemic LF (hereafter LF method); and 2) a statistical comparison (similar to Kerber \& Santiago 2005) between observed and model CMDs (hereafter CMD method), where $\alpha$ is a free input parameter in the CMD modelling process. The main difference between the two methods is that the CMD method potentially uses all the information contained in the CMD, including the effects of unresolved binaries and photometric uncertainties.

The paper is divided as follows. In Sect. 2 we present a brief description of our data and the physical properties of the clusters. In Sect. 3 we present the two methods of determining the PDMF slope and their results. These results are then discussed in Sect. 4, where we also compare them with those available in
Table 1. Main parameters of the clusters in the sample.

\begin{tabular}{lccccccc}
\hline \hline Cluster & $N_{\text {clus }}$ & $\begin{array}{c}R_{\text {core }} \\
\left({ }^{\prime \prime}\right)\end{array}$ & $\begin{array}{c}\text { max } \\
\left({ }^{\prime \prime}\right)\end{array}$ & $Z$ & $\log (\tau / \mathrm{yr})$ & $(m-M)_{0}$ & $E(B-V)$ \\
\hline NGC 1805 & 2564 & 5.5 & 55 & 0.008 & $7.80^{*}$ & 18.55 & 0.03 \\
NGC 1818 & 3929 & 10.1 & 60 & 0.004 & $7.80^{*}$ & 18.45 & 0.00 \\
NGC 1831 & 7136 & 18.3 & 90 & 0.012 & 8.70 & 18.70 & 0.00 \\
NGC 1868 & 5675 & 6.7 & 70 & 0.008 & 8.95 & 18.70 & 0.00 \\
Hodge 14 & 1196 & 7.4 & 40 & 0.006 & 9.25 & 18.55 & 0.03 \\
\hline
\end{tabular}

* Youngest isochrone available by Girardi et al. (2000).

the literature. Finally, in Sect. 5 we present a summary and our concluding remarks.

\section{The data}

We used data taken with HST/WFPC 2 as part of a cycle 7 project entitled "Formation and evolution of rich LMC clusters" (Beaulieu et al. 1999). For each cluster and a nearby field, images were obtained using the $F 555 W(\sim V)$ and $F 814 W(\sim I)$ broad band filters. A detailed description of the photometry and sample completeness corrections can be found in Santiago et al. (2001) and Castro et al. (2001). Kerber \& Santiago (2005) made a detailed study of the resulting CMDs in the central cluster regions, in order to infer the cluster's global parameters, such as age, metallicity, foreground extinction, and distance. Their work was based on a detailed CMD modelling process. In brief, the modelling is based on the generation of synthetic CMDs to be compared to the observed one. For a realistic comparison to the models, the observed CMDs had to be corrected for selection effects, such as photometric incompleteness and fieldstar contamination. Random photometric uncertainties in the real data were also quantified and applied to the model CMDs. Finally, different statistical tools were used in the model vs. data CMD comparison.

Table 1 lists the main parameters of the clusters in the sample. The cluster name is followed by the total number of stars $\left(N_{\text {clus }}\right)$ in its final CMD after being corrected for the effects mentioned earlier (see Kerber \& Santiago 2005 for details). This table also lists the cluster's adopted core radius ( $R_{\text {core }}$, as determined by Mackey \& Gilmore 2003); and the radius at which the star density falls to the field density $\left(R_{\max }\right)$. The metallicity $(Z)$, logarithmic age $(\tau)$, intrinsic distance modulus $\left((m-M)_{0}\right)$, and reddening value $(E(B-V))$ were kept fixed in the PDMF analysis and are consistent with the ones determined by Kerber \& Santiago (2005).

\section{Analysis}

The PDMF is considered here to be a power law:

$\xi(m)=\frac{\mathrm{d} N}{\mathrm{~d} m}=\xi_{0} m^{-\alpha}$

where $\xi_{0}$ is a normalization constant and $\alpha$ the PDMF slope. Different parameterizations are found in the literature, often making use of multiple power laws. Given the limited mass range of main-sequence stars in the CMDs studied here, use of a single slope is justified.

To analyse the positional dependence of $\alpha$ within a cluster, we divided its CMD stars into subsamples, according to the distance from the cluster centre, defining several annuli. The inner and outer radial limits of each ring were chosen to ensure that each subsample would typically contain 600 stars, allowing 
a statistically significant number of stars to contribute to each PDMF determination.

To determine the PDMF slope $(\alpha)$ in each ring we used two distinct approaches, each one made up of several steps:

1. derivation of the systemic LF; conversion of the LF into a PDMF, according to a given mass-luminosity relation; a power law fit to the PDMF obtained from the systemic LF We refer to this method as the LF method;

2. generation of synthetic CMDs with different input PDMFs, keeping the binary fraction and model isochrone parameters fixed; statistical comparison between observed and model CMDs; determination of the best-fitting CMD models for each data CMD. In this case, the PDMF slope $\alpha$ is a free input parameter of the CMD models. As the statistical comparison in this case makes use of the information available in the entire CMD plane, we call it the CMD method.

Both approaches require conversion from mass to luminosity (CMD method) or vice-versa (LF method). The massluminosity relation used in these conversions is provided by Padova isochrones (Girardi et al. 2000) that are shifted in magnitude and colour by $(m-M)_{0}$ and $E(B-V)$ and where the parameters (including $Z$ and $\tau$ ) are as given in Table 1 . When necessary, we interpolated the original Padova isochrone grid in metallicity (see Kerber \& Santiago 2005, Sect. 3.2 and their Fig. 13) in order to generate an isochrone with the quoted values.

Each approach displays its own advantages and disadvantages. The LF method, by construction, does not use all the twodimensional information contained in the CMD plane. This may actually be considered an advantage, since the PDMF can be reliably recovered with a smaller number of stars, as each magnitude bin concentrates the information spread along the MS colour width. On the other hand, the effect caused by unresolved binaries may be crucial, since the CMD position of primary stars will be spread redwards and brightwards due to the presence of the secondaries. If unaccounted for, unresolved binarity will cause some shallowing in the recovered PDMF, as the system masses resulting from their combined luminosities will be larger than the masses of the individual components.

Several previous works have taken the effect of unresolved binaries into account in the recovered PDMF or IMF from a systemic LF. Kroupa et al. (1991) have managed to reconcile the LF of local volume-limited samples of Galactic field stars with the LF inferred from photometric surveys of more distant by correcting them for the systematics caused by unresolved pairs. More recently, Kroupa (2001) showed that the single-star IMFs can be systematically steeper by 0.5 between $0.1 \leq m \leq 1.0 M_{\odot}$ than the Galactic-field IMF. Studying LMC star clusters, Sagar $\&$ Richtler (1991) determined that the recovered PDMF from the systemic LF can become significantly steeper (by $\Delta \alpha \simeq 1.0$ ) if the binary fraction is large $\left(f_{\text {bin }} \gtrsim 0.50\right)$ and $\alpha \sim 0.5$. Similar techniques were applied by Sandhu et al. (2003) to correct the PDMF for intermediate/old open clusters.

In order to simulate the effect of unresolved binaries in PDMF determination we introduced it in the CMD modelling process. The well-known signature of unresolved pairs in a CMD (see Hurley \& Tout 1998 for a demonstration of the effect) can be modelled in a straightforward manner by applying the combined fluxes and colours from two stars to a given fraction $\left(f_{\text {bin }}\right)$ of systems. One caveat may be the uncertainties in the distribution $(\mathrm{d} N / \mathrm{d} q)$ of secondary/primary mass ratios ( $q=m_{2} / m_{1} \leq 1.0$, where $m_{1}$ and $m_{2}$ are, respectively, the primary and secondary masses). This may at first sight be regarded as an extra degree of freedom in the modelling process, since secondary star masses may not necessarily be drawn from the same distribution as the primary stars. However, this possibility poses a question of what a mass function is meant to be, as it would not be uniquely defined even in a single population. Here we adopt the assumption that secondary stars in binary systems have masses drawn from the same PDMF as primary stars or as single stars. To maximize the possible effect of unresolved binaries and therefore explore the most of this effect, we used $f_{\text {bin }}=100 \%$.

Furthermore, the CMD modelling naturally incorporates the photometric uncertainties into the PDMF determination, and can potentially incorporate other observational effects that may influence the conversion of magnitude and colour information into mass. The major disadvantage of a CMD method is the prior PDMF parameterization, here modelled as a power law with one free-parameter for $m \geq 0.80 M_{\odot}$ ( $\gtrsim$ observed lower-mass limit). Even though it does not directly affect the PDMF in the observed regime, we fixed a shallow slope $\alpha=1.30$ in the lower mass range $\left(0.08 \leq m / M_{\odot} \leq 0.80\right)$. This is consistent with the IMF proposed by Kroupa (2002) in this mass range. This relatively shallow slope in the low-mass range also yields an enhanced effect of unresolved binaries. Although in our simulations all stars have one companion, only pairs with $q \gtrsim 0.60$ will significantly change the CMD position of the primary star. The fraction of such effective binary systems, $f_{\text {bin,eff }}$, is $\sim 20 \%$. They are practically the only ones responsible for the effect that unresolved binaries may cause in the CMD or in the LF. We refer to Tout (1991) for a demonstration of the sensitivity of PDMF slope with the mass-ratio distribution in the low-mass regime.

\subsection{LF method}

Mass segregation was observed by Santiago et al. (2001) in their study of the LFs of the clusters in our sample. Then de Grijs et al. (2002a) converted LFs into PDMFs and presented their positional dependence for the younger clusters, NGC 1805 and NGC 1818. But those authors did not use CMD modelling techniques to investigate the behaviour of $\alpha$ as a function of distance from the cluster centre. Also, the mass-luminosity relations used in previous works were not the same as those used here, which are based on careful constraints on the cluster's ages and metallicities, which come from our previous CMD modelling.

Notice that the mass-luminosity relation is a key ingredient in conversion from an LF into a PDMF, a traditional procedure widely used in stellar statistics. By definition, the $\operatorname{LF}\left(\Phi\left(M_{555}\right)\right)$ in the $M_{555}$ absolute magnitude is given by

$\Phi\left(M_{555}\right)=\frac{\mathrm{d} N}{\mathrm{~d} M_{555}}$

where $\mathrm{d} N$ is the number of individual stars that have absolute magnitude inside $\left(M_{555}, M_{555}+\mathrm{d} M_{555}\right)$.

If the PDMF is expressed in linear mass bins $(\xi(m))$, it is related to the LF as follows

$\xi(m)=\frac{\mathrm{d} N}{\mathrm{~d} m}=-\Phi\left(M_{555}\right)\left[\frac{\mathrm{d} m\left(M_{555}\right)}{\mathrm{d} M_{555}}\right]^{-1}$

where the $m\left(M_{555}\right)$ is the mass-luminosity relation. Here one clearly sees the importance of the choice of suitable stellar evolution models, as they provide not only the mass-luminosity relation, but also its derivative. As previously discussed, it is important to keep in mind that the observed photometric data 


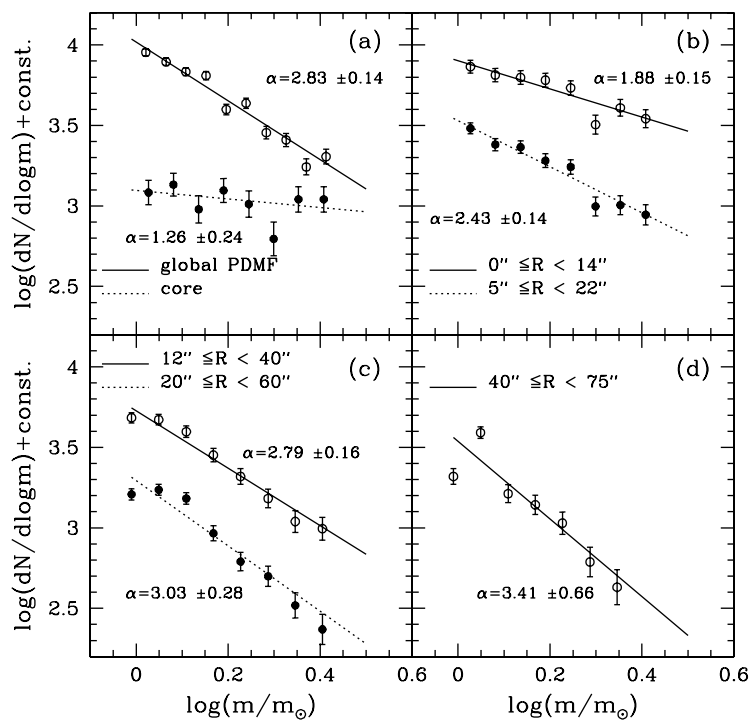

Fig. 1. PDMFs at different concentric regions around NGC 1805. The lines are power law fits to the PDMFs. The slope values and their corresponding fit errors are given in each case, as well as the inner and outer radii of each region.

suffer from unresolved binarity and therefore allow us to construct only the systemic LF. As a consequence, there is not a unique mapping from one $M_{555}$ magnitude to one stellar mass; the LF method may be seen as a first attempt to recover the PDMF. We refer the reader to Sect. 4.1, where we present an approach to evaluating the effect of unresolved pairs in the recovered PDMF from a systemic LF.

Analogous to the LF, the PDMF may also be expressed in logarithmic $\left(\log _{10}\right)$ mass bins $\left(\xi_{\mathrm{L}}(m)\right)$, in which case it is related to $\xi(m)$ by

$\xi_{\mathrm{L}}(m)=\frac{\mathrm{d} N}{\mathrm{~d} \log m}=\xi(m)\left[\frac{\mathrm{d} \log m}{\mathrm{~d} m}\right]^{-1}=\log e m \xi(m)$.

Also parameterizing the logarithmic PDMF as a power law, we have $\xi_{\mathrm{L}}(m)=\xi_{\mathrm{L}, 0} m^{\Gamma}$, whose slope $\Gamma$ is correlated with $\alpha$ by $\Gamma=-(\alpha-1)$.

Taking the logarithm on both sides of Eq. (1), we have $\log \xi=-\alpha \log m+\log \xi_{0}$ or $\log \xi_{\mathrm{L}}=-(\alpha-1) \log m+\log \xi_{0}-0.362$. In other words, in a log-log plot, both the linear and logarithmic power law PDMFs should behave as a straight line, whose slope yields $\alpha$ directly.

Figures 1-5 present the behaviour of the PDMFs recovered directly (without any treatment of the unresolved binarity) from the systemic LFs at different concentric regions around each cluster. For convenience, we determine $\xi_{\mathrm{L}}$ and plot it in a logarithmic scale, where we shift the values of $\log \left(\xi_{\mathrm{L}}\right)$ up or down to avoid overlapping in the panels. In each case $\alpha$ was obtained by means of a linear fit, shown as a straight line in the figure panels. Panel a) shows the PDMF for all stars in each cluster and the PDMF for the stars within the core. In all cases $\alpha$ is smaller in the core than for the entire cluster, which indicates mass segregation. Besides, these figures show the expected signature of mass segregation: the PDMF is shallower (smaller $\alpha$ ) in the inner regions (panel b) than in the outer regions (panel d). Also, in general, the single slope fit is a very adequate description of the data. The exceptions to this rule often result from a sudden drop at the low-mass end $\left(\log \left(m / M_{\odot}\right) \sim 0.0, m \sim 1.0 M_{\odot}\right)$ of the PDMFs in the central regions of the richest clusters, such as

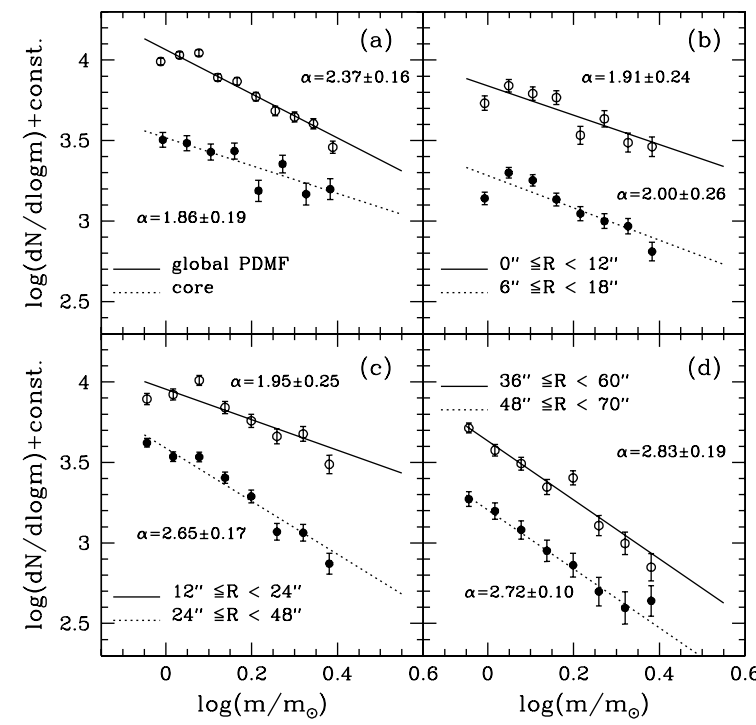

Fig. 2. Same as in Fig. 1, but now for NGC 1818.

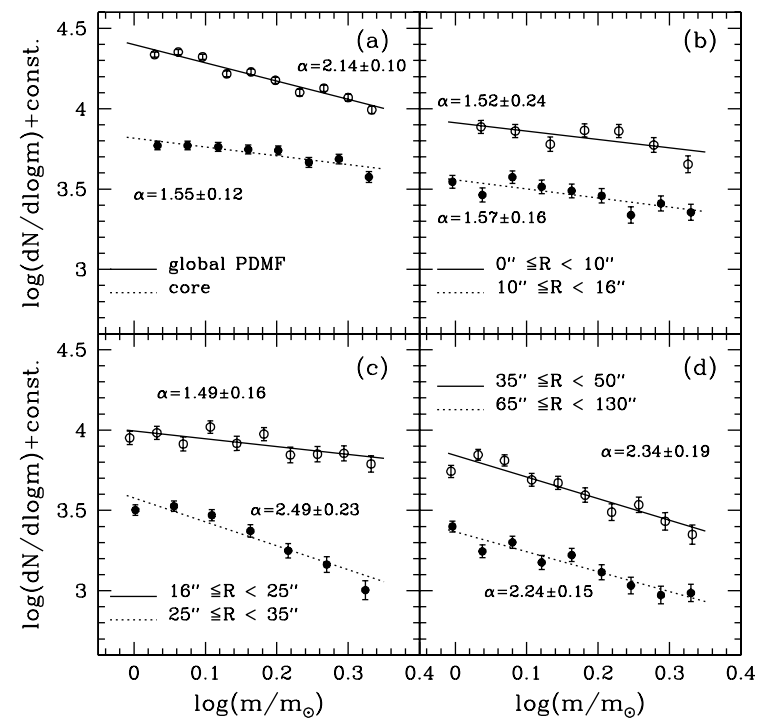

Fig. 3. Same as in Fig. 1, but now for NGC 1831.

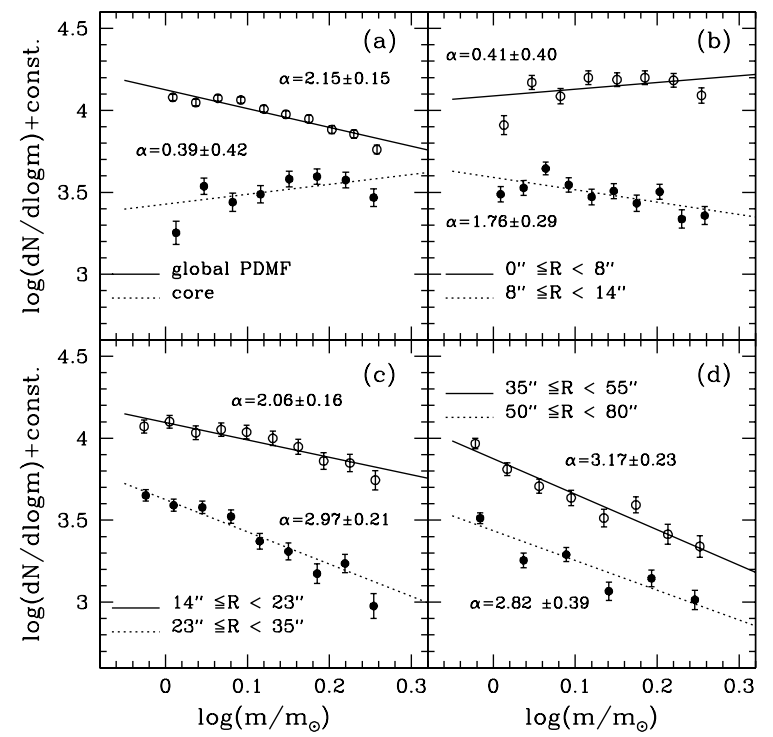

Fig. 4. Same as in Fig. 1, but now for NGC 1868. 


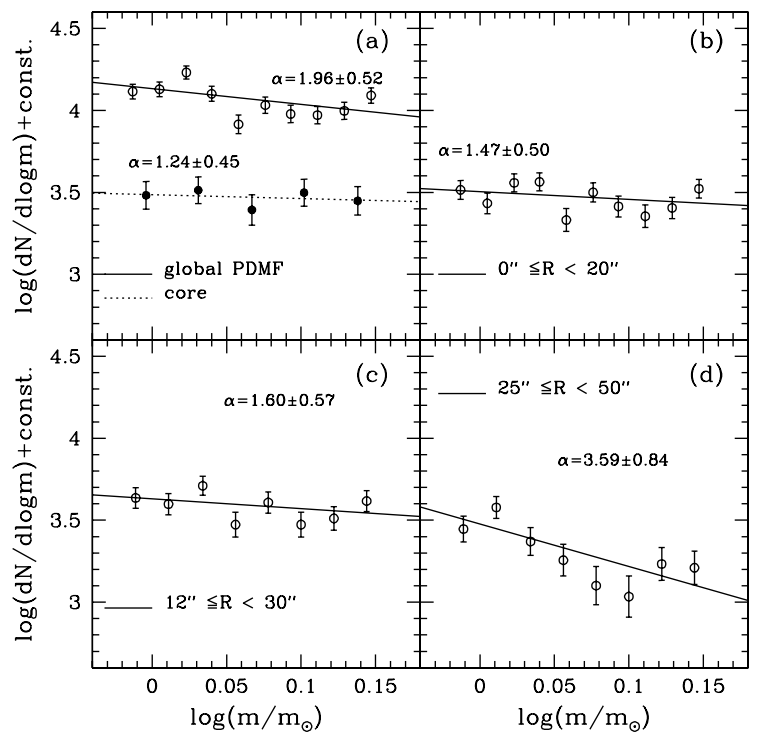

Fig. 5. Same as in Fig. 1, but now for Hodge 14.
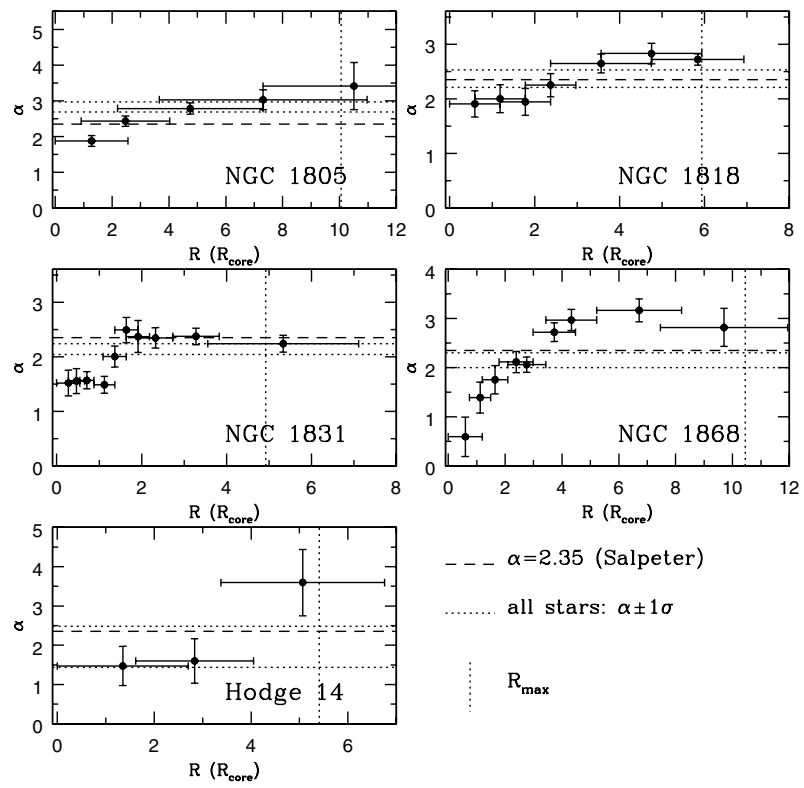

$---\alpha=2.35$ (Salpeter)

........ all stars: $\alpha \pm 1 \sigma$ $R_{\max }$

Fig. 6. The PDMF slope vs. $R$ inferred from the LF analysis. The horizontal dotted lines show the $2 \sigma$ range in the global PDMF slope. The horizontal dashed line is the reference Salpeter value $(\alpha=2.35)$. The $R_{\max }$ is marked as the vertical line.

NGC 1868. These deviations from a power law are very likely due to residuals in the correcting for incompleteness effects.

Figure 6 plots $\alpha$ as a function of distance $R$ from the cluster centre. This distance is expressed in units of the core radius, listed in Table 1 . The effect of mass segregation is again clearly seen for all clusters. The vertical bars on each point represent the linear-fit uncertainties in $\alpha$. The horizontal bars just show the limits of the annuli. Some concentric regions are partially overlapping in order to yield a more continuous behaviour of the PDMF slope and to increase the statistical significance in each PDMF determination. The dotted-horizontal lines constrain the $1 \sigma$ range of $\alpha$ fitted to the global PDMF. This range can be compared with the Salpeter (1955) value $(\alpha=2.35)$, marked as a dashed-horizontal line. In all the others clusters, besides Hodge $14, \alpha(R)$ has a Salpeter value around $R \simeq 2-3 R_{\text {core. }}$. In the outer regions, the relation $\alpha$ vs. $R\left(R_{\text {core }}\right)$ flattens for NGC 1818 ,
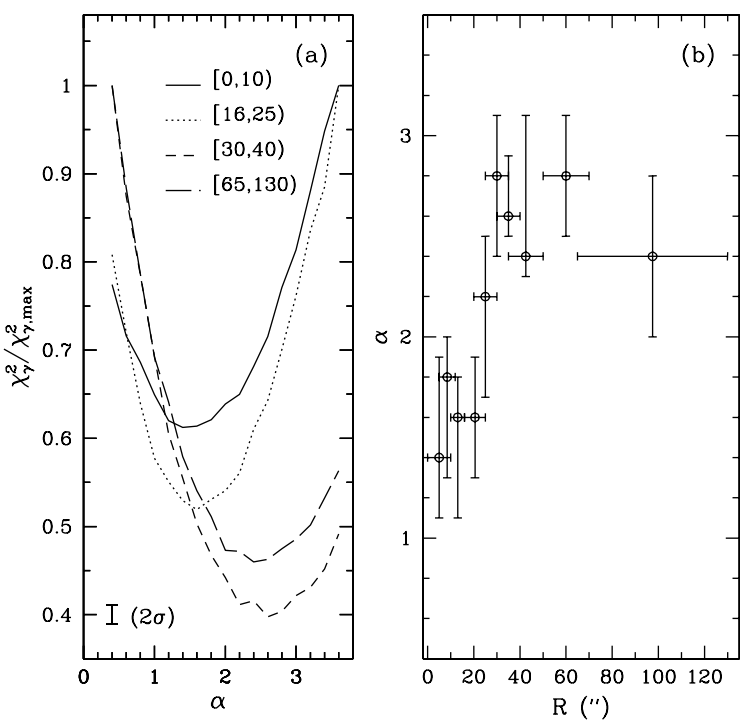

Fig. 7. The CMD method for NGC 1831. Panel a) illustrates how the statistic $\chi_{\gamma}^{2}$ varies with the adopted $\alpha$ used in the CMD models at different concentric annuli. Panel b) shows the best-fit $\alpha$ vs. $R$ relation.

NGC 1831, and NGC 1868. This may be the result of the dynamical loss of lower-mass stars from the clusters.

\subsection{CMD method}

Only the systemic LF, which suffers from the effect unresolved binaries, is directly extracted from the data, as discussed in the introduction to this section. Therefore, we deal in this section with the 2D CMD modelling process, an approach that is capable of explicitly taking unresolved binarity into account.

Our CMD modelling process and the statistical techniques of CMD comparisons were extensively explained in Kerber \& Santiago (2005) and Kerber et al. (2002). Here we only underline some important aspects. The modelling process assumes that the cluster is a single stellar population (SSP) that generates a synthetic main-sequence (MS) in the CMD plane, where we introduce as model inputs the information about metallicity $(Z)$, age $(\tau)$ (given by a Padova isochrone; Girardi et al. 2000), intrinsic distance modulus $\left((m-M)_{0}\right)$, reddening value $(E(B-V))$, PDMF slope $(\alpha)$, and fraction of unresolved binaries $\left(f_{\text {bin }}\right)$. By exploring a regular model grid, we may then find the best models by means of statistical comparisons carried out in 1 and 2 dimensions and, therefore, the physical parameters that best constrain the cluster CMD. By modelling the CMDs in the central region, Kerber \& Santiago (2005) inferred $Z,(m-M)_{0}$, and $E(B-V)$ for each cluster. They also determined the age of three clusters: NGC 1831, NGC 1868, and Hodge 14. A set of values consistent with these determination is listed in Table 1.

We here model the CMDs separated by annuli in order to investigate the positional dependence of the only remaining free parameter: $\alpha$. Figure 7 illustrates the CMD method for NGC 1831. Panel a) shows how the statistic $\chi_{\gamma}^{2}$ varies as a function of the input value of $\alpha$ used to build the synthetic CMDs. Each curve represents the run of $\chi_{\gamma}^{2}$ (normalised by its maximum value, $\chi_{\gamma, \text { max }}^{2}$ ) with $\alpha$ at a given cluster ring. The effect of mass segregation is already clear in this figure, as the minimum value $\chi_{\gamma, \min }^{2}$ occurs at higher $\alpha$ as the outer annuli are considered, reflecting a gradual steepening in the PDMF.

In order to determine $\alpha$ and its associated uncertainty from this method, 100 realisations of the model that yielded $\chi_{\gamma, \min }^{2}$ 

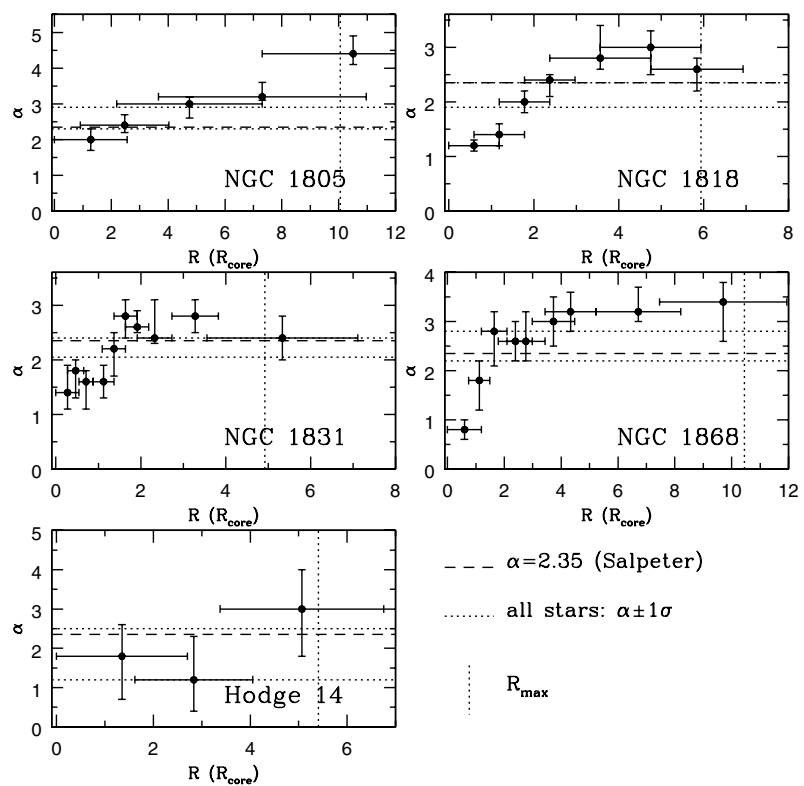

- $-\alpha=2.35$ (Salpeter)

........ all stars: $\alpha \pm 1 \sigma$

$R_{\max }$

Fig. 8. The PDMF slope vs. $R$ in the 2D CMD analysis. The lines are the same as in the Fig. 6.

were run, from which a dispersion in $\chi_{\gamma}^{2}$ for this model, $\sigma_{\chi}$, was determined. Therefore, the models that satisfy the criterion

$\chi_{\gamma}^{2} \leq \chi_{\gamma, \min }^{2}+\sigma_{\chi}$

are statistically of similar quality as the one that yields the minimum. The average value of $\alpha$ for these models is then considered as the one that best describes the PDMF, and the standard deviation around this average represents its uncertainty. Finally, panel b) in Fig. 7 shows the $\alpha$ values determined for all annuli, again revealing variations with position inside the cluster.

Following this procedure for all clusters, we built Fig. 8, the CMD method counterpart of Fig. 6. Again all clusters show evidence of mass segregation. After taking the uncertainties into account, these results are in very good agreement with those from the LF method.

\section{Discussion}

As shown in the previous section the spatial dependence of $\alpha$ clearly reveals the presence of mass segregation in all clusters. Except in some cases where uncorrected for incompleteness effects at lower masses may be strongly affecting the PDMF slope, the results in both LF and CMD methods used here are in clear agreement and within the uncertainties.

Previous, detailed spatial determinations of the PDMF slope were made only for NGC 1805 (de Grijs et al. 2002a) and NGC 1818 (de Grijs et al. 2002a; Gouliermis et al. 2005). In both cases these authors apply the LF method to their HST/WFPC2 data to determine the $\Gamma(=-\alpha+1)$ behaviour with distance to the cluster centre. In general our results agree with theirs, even in the case where the data are restricted to a higher stellar mass range ( $m \gtrsim 2.0 M_{\odot}$ ) (Gouliermis et al. 2005). For the other clusters, there are no analyses of similar quality and mass range in the literature.

Notice that $\alpha \sim 2.35$ in the interval $2<R / R_{\text {core }}<3$ for all clusters, except for Hodge 14 , for which $\alpha \sim 2.35$ at $R / R_{\text {core }} \simeq 4$. This range is close to the clusters' half-light (or mass) radii.

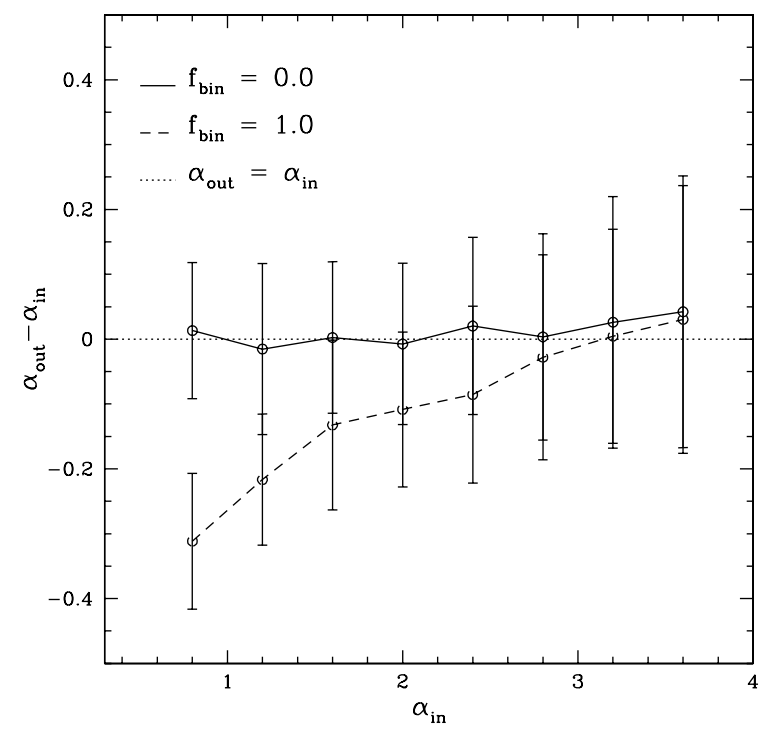

Fig. 9. Control experiment to determine the effect of unresolved binaries in the PDMF slope recovered from a systemic LF. In the vertical axis we show the difference between the recovered slope $\alpha_{\text {out }}$ and the input slope $\alpha_{\text {in }}$. Solid line (dashed line): LF generated with $f_{\text {bin }}=0 \%\left(f_{\text {bin }}=\right.$ $100 \%)$.

\subsection{The unresolved binarity effect}

The major systematic effect over the usual LF method to determine the PDMF slope is the one caused by unresolved binaries; a flatter PDMF (lower $\alpha$ ) is likely to be recovered if binaries are not accounted for (see the discussion in Sect. 3). In order to assess this issue directly in our approaches, we generate control experiments where we simulate artificial CMDs with $f_{\text {bin }}=100 \%$ and an input PDMF slope $\left(\alpha_{\text {in }}\right)$ and then recover the PDMF slope $\left(\alpha_{\text {out }}\right)$ by applying the LF method. As discussed in Sect. 3, although the primary and secondary stellar masses were drawn from the same PDMF, only primary stars with mass $\gtrsim 0.9 M_{\odot}$ were directly taken into account, in accordance with the fainter magnitude limit in the observed data. Furthermore, random pairing and $\alpha=1.30$ for $0.08 \leq m / M_{\odot} \leq$ 0.8 (unseen mass regime) yield $f_{\text {bin,eff }}(q \geq 0.60) \sim 0.20$. The results of these experiments are shown in Fig. 9. As expected, the recovered PDMF is flatter than the input one, and the amplitude of this effect is greater for flatter input PDMFs. Except for $\alpha_{\text {in }}<1.2$, however the amplitude of $\alpha_{\text {out }}-\alpha_{\text {in }}$ is smaller than the uncertainties in $\alpha_{\text {out }}$. Thus, for an unresolved binary fraction consistent with the one used in our CMD modelling process, its effect in the derived PDMF seems not to play a decisive role.

\subsection{Dynamical mass segregation inside the core?}

To get some clues about the nature of the mass segregation, we evaluated the two-body relaxation time using the following expression (Binney \& Tremaine 1987):

$t_{\mathrm{rl}}=\frac{6.5 \times 10^{8}}{\ln (0.4 N)}\left(\frac{M}{10^{5} M_{\odot}}\right)^{1 / 2}\left(\frac{M_{\odot}}{m_{*}}\right)\left(\frac{R}{\mathrm{pc}}\right)^{3 / 2}$

where $N$ and $M$ are the total number of stars and the total mass inside some radius $R$, respectively, and $m_{*}$ is a characteristic stellar mass. This is the time needed for stellar encounters to redistribute the stellar kinetic energy so that the the velocity distribution is approximately Maxwellian. However, it is important to keep in mind that this expression comes from analytical 
Table 2. Dynamical parameters and timescales estimated for the region inside the core radius.

\begin{tabular}{lrrrrr}
\hline \hline Cluster & $N_{\text {obs }}$ & $\begin{array}{r}N \\
\left(\times 10^{3}\right)\end{array}$ & $\begin{array}{r}M \\
\left(\times 10^{3} M_{\odot}\right)\end{array}$ & $\begin{array}{r}t_{\mathrm{rl}} \\
(\mathrm{Myr})\end{array}$ & $\tau / t_{\mathrm{rl}}$ \\
\hline NGC 1805 & 66 & $3.9 \pm 0.5$ & $2.2 \pm 0.3$ & $16 \pm 4$ & $0.64 \pm 0.10$ \\
NGC 1818 & 140 & $7.5 \pm 0.7$ & $4.1 \pm 0.4$ & $48 \pm 7$ & $0.51 \pm 0.10$ \\
NGC 1831 & 464 & $38.4 \pm 1.9$ & $20.9 \pm 1.2$ & $222 \pm 19$ & $2.26 \pm 0.10$ \\
NGC 1868 & 182 & $16.5 \pm 1.3$ & $8.9 \pm 0.8$ & $35 \pm 5$ & $25.43 \pm 1.00$ \\
Hodge 14 & 53 & $3.7 \pm 0.5$ & $2.1 \pm 0.3$ & $24 \pm 5$ & $76.25 \pm 4.00$ \\
\hline
\end{tabular}

considerations. The $t_{\mathrm{rl}}$ obtained here allows a simple estimate of dynamical age for each cluster, since a more realistic treatment is only obtained through $N$-body simulations (Kroupa et al. 2001; Baumgardt \& Makino 2003).

As discussed by Stolte et al. (2002), using the present physical conditions of a cluster can only lead to the current dynamical status. We therefore decided to evaluate $t_{\mathrm{rl}}$ for approximated initial conditions, where we take the IMF as that from Kroupa (2002) $\left(\alpha=2.30\right.$ for $0.50<m / M_{\odot} \leq 120$ and $\alpha=1.30$ for $0.08<m \leq 0.50 M_{\odot}$ ) normalised by the number of observed stars in a bright magnitude (massive) range. This range corresponds to $21.0 \leq V_{555} \leq 20.0\left(1.5 \lesssim m / M_{\odot} \lesssim 2.0\right)$ for NGC 1805, NGC 1818, NGC 1831, and NGC 1868, and $22.0 \leq$ $V_{555} \leq 21.0\left(1.2 \lesssim m / M_{\odot} \lesssim 1.5\right)$ for Hodge 14 . The characteristic stellar mass $m_{*}$ was taken to be the median mass for the adopted IMF inside the observed magnitude range, $m_{*}=$ $1.40 M_{\odot}$. Table 2 shows, for all clusters, the physical parameters estimated using this approach for all stars $\left(0.08 \leq m / M_{\odot} \leq\right.$ 120 ) inside the core radius. The uncertainties in $N$ (Col. 3 ), $M$ (Col. 4), and $t_{\mathrm{rl}}$ (Col. 5) result from the statistical fluctuations in the observed star counts $\left(N_{\text {obs }}\right)$ (Col. 2) used in the IMF normalization.

In this table we also present the ratio $\tau / t_{\mathrm{rl}}$ in order to give an idea of the dynamical age of each cluster. The uncertainties in this ratio were propagated from the corresponding uncertainties in $\tau$ and $t_{\mathrm{rl}}$. This ratio spans a wide range of values (0.2 to 30). The relation between this parameter, evaluated for the core radius, and the corresponding PDMF slope (determined only by the LF method due to low statistics) is plotted in Fig. 10. Although strongly based on the NGC 1868 result, the relation suggests that dynamically younger clusters tend to have steeper PDMF than dynamically older ones, as expected from the effects of dynamical mass segregation. We fit a linear relation (dashed line) between these parameters (correlation coefficient (c.c.) $=0.64)$, with slope $\mathrm{d} \alpha_{\text {core }} / \mathrm{d} \log \left(\tau / t_{\mathrm{rl}}\right)=-0.38 \pm 0.30$. Also note that all PDMF are flatter than Salpeter (marked as a horizontal dotted line in this figure). By our fit one could expect that only clusters with $\log \left(\tau / t_{\mathrm{rl}}\right) \sim-1.5$ would have a PDMF with a Salpeter slope within their cores.

On the other hand, if NGC 1868 is omitted from the linear relation (c.c. $=0.47)$, the slope becomes flatter $(-0.20 \pm 0.27)$. The lack of a strong trend in the central $\alpha$ value with age could mean that mass segregation takes place at the onset of star formation within a cluster (primordial segregation) or on such a short timescale that it leaves little room for subsequent mass segregation due to dynamical effects on longer timescales. There is no specific work applying $N$-body simulations to address how fast the PMDF evolves in a typical globular cluster centre; in less dense environments, Kroupa et al. (2001) have shown that the mass segregation in the ONC can be explained as a dynamical effect. Notice also that there is a clear PDMF flattening with dynamical age inside the core radius of open clusters (Bonatto \& Bica 2005).

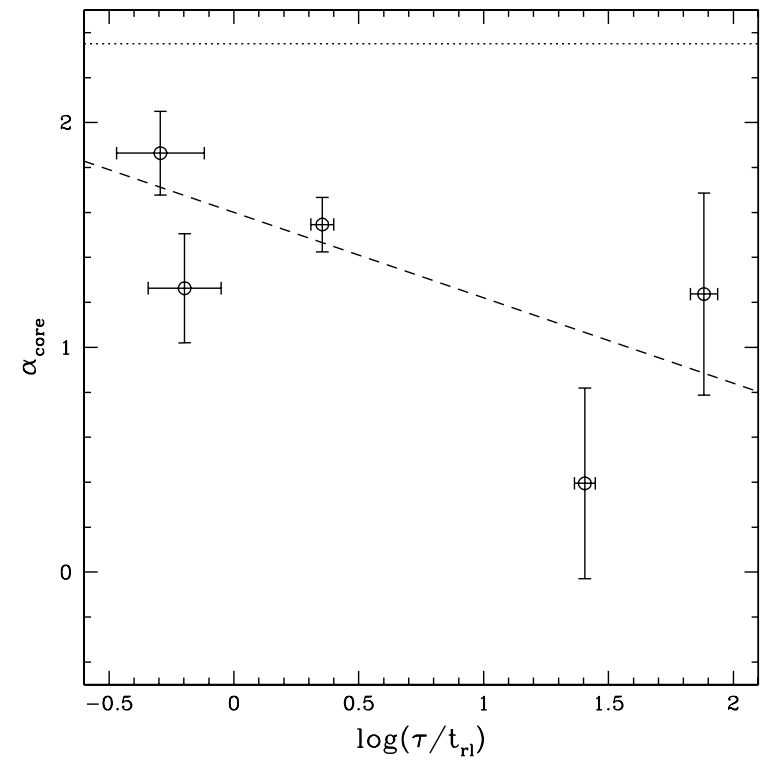

Fig. 10. The PDMF slope vs. $\log \left(\tau / t_{\mathrm{rl}}\right)$ relation inside the core radius.

Table 3. Estimates of the global dynamical parameters and timescales for the clusters.

\begin{tabular}{lrrrrr}
\hline \hline Cluster & $n_{\mathrm{obs}}$ & $\begin{array}{r}n_{\text {tot }} \\
\left(\times 10^{3}\right)\end{array}$ & $\begin{array}{r}m_{\text {tot }} \\
\left(\times 10^{3} M_{\odot}\right)\end{array}$ & $\begin{array}{r}t_{\mathrm{rl}} \\
(\mathrm{Gyr})\end{array}$ & $\tau / t_{\mathrm{rl}}$ \\
\hline NGC 1805 & 471 & $30.7 \pm 1.7$ & $16.8 \pm 0.9$ & $1.1 \pm 0.1$ & $0.010 \pm 0.002$ \\
NGC 1818 & 774 & $46.3 \pm 2.1$ & $25.2 \pm 1.3$ & $1.4 \pm 0.1$ & $0.018 \pm 0.002$ \\
NGC 1831 & 1539 & $109.4 \pm 3.5$ & $59.5 \pm 2.1$ & $3.7 \pm 0.2$ & $0.135 \pm 0.010$ \\
NGC 1868 & 929 & $63.9 \pm 1.3$ & $34.8 \pm 1.5$ & $2.0 \pm 0.1$ & $0.442 \pm 0.020$ \\
Hodge 14 & 317 & $22.9 \pm 1.5$ & $12.5 \pm 0.8$ & $0.6 \pm 0.1$ & $3.073 \pm 0.150$ \\
\hline
\end{tabular}

\subsection{Dynamical origin for the loss of lower-mass stars?}

In order to investigate a potential dynamical origin of the loss of lower-mass stars, we evaluated the same quantities as in the previous section, but considering all stars in each cluster. The results are presented Table 3 . Although $t_{\mathrm{rl}}$ does not correspond exactly to the timescale for stellar evaporation, it is expected to scale with this one (Binney \& Tremaine 1987). As one can see in the last column of Table 3 , the $\tau / t_{\mathrm{tl}}$ values again span a wide range $\left(4 \times 10^{-3}\right.$ to 1.2$)$. The relation between global $\alpha$ (from LF and CMD methods) and global $\log \left(\tau / t_{\mathrm{tl}}\right)$ is plotted in Fig. 11. For both methods there is a trend in the sense that dynamically older clusters again have lower values of $\alpha$. Using the results of the LF method, we again fit a linear relation (shortdashed line, c.c. $=0.87$ ) between these parameters and find a slope $\mathrm{d} \alpha_{\text {global }} / \mathrm{d} \log \left(\tau / t_{\mathrm{rl}}\right)=-0.37 \pm 0.12$. We also fit a linear relation using the results of the CMD method (long-dashed line, c.c. $=0.50)$ and find a slope of $-0.13 \pm 0.13$.

It is very interesting to notice that this trend is consistent with recent results in the literature for a variety of systems. Using $N$ body simulations, Baumgardt \& Makino (2003) reproduce the observed shallowing in the global PDMF of globular clusters and attribute this effect to the lower-mass stars preferentially depleted from the cluster due to dynamical mass segregation. They also find that the details of this process are nearly independent of the starting conditions. Bonatto \& Bica (2005), analysing 2MASS data for 11 open clusters, clearly show that these systems also have the same effect. In both cases the MF flattening reaches at least 0.8 in slope, again consistent with what we observe in the rich LMC clusters. Durgapal \& Pandey (2001) show that intermediate age and old open clusters tend to have a 


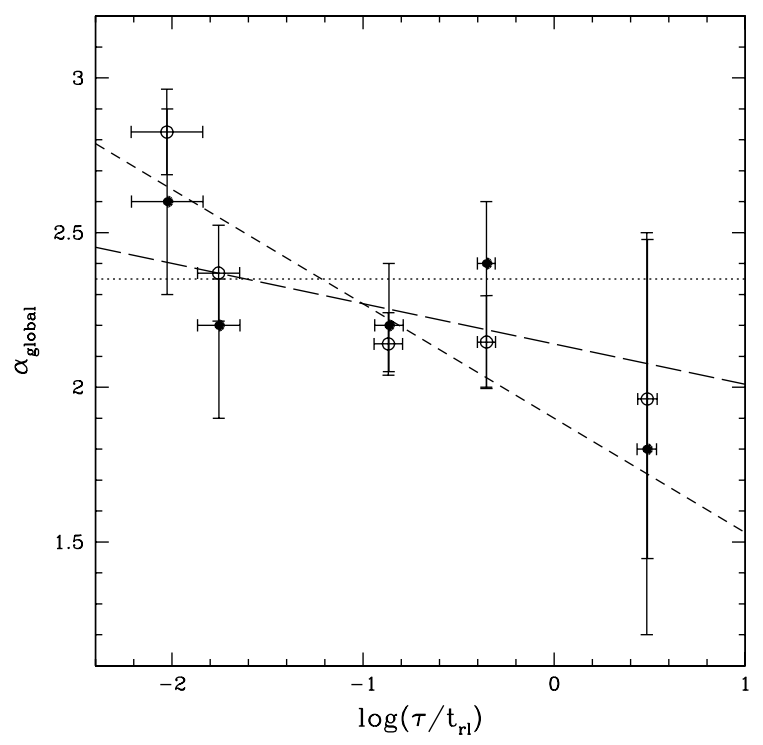

Fig. 11. The PDMF slope vs. $\log \left(\tau / t_{\mathrm{rl}}\right)$ for the global cluster. Open (filled) circles correspond to LF (CMD) method. The best linear fit for the LF (CMD) method is shown as a short(long)-dashed line. The dotted line corresponds to the Salpeter slope.

smaller ratio between their present radius and their limiting radius as they become older. They attribute this to the effect of loss of stars.

\section{Summary and conclusions}

We have analysed the shape of the PDMF at different radii for a sample of 5 rich LMC clusters. The data are the result of deep imaging with HST/WFPC2. The PDMFs were determined by two distinct approaches: 1) a traditional method of converting stellar luminosities into masses; 2) a full modelling of the colour-magnitude diagrams of the clusters, accounting for the effects of unresolved binaries and photometric errors. Our results hold for both approaches and are insensitive to the details of how the PDMF and its slope are determined. Control experiments reveal that the unresolved binaries flatten the PDMF recovered by the traditional methods. However this effect is less than the uncertainties in the $\alpha$ determination.

We found significant mass segregation in all of them. The effect is expected in the sense that the PDMF is steeper further out than in the core: $\alpha \lesssim 1.80$ for $R \leq 1 R_{\text {core }}$ and $\alpha \sim$ Salpeter (=2.35) inside $R=2 \sim 3 R_{\text {core }}$ (except for Hodge 14, where it occurs at $\left.R \sim 4 R_{\text {core }}\right)$. Since the global PDMF is also near Salpeter, we confirm previous claims that the PDMF evaluated around the half-mass radius is consistent with the global PDMF (Kroupa 2002).

The spatial dependence of the PDMF slope was previously presented by de Grijs et al. (2002a) for the youngest two clusters in our sample, NGC 1805 and NGC 1818, and by Gouliermis et al. (2005) for NGC 1818.

We have investigated the origin of mass segregation and the loss of lower-mass stars by inferring a dynamical age $\left(\tau / t_{\mathrm{rl}}\right)$ for each cluster. This was done in two regions: inside the cluster core and for the entire system. In both cases we notice that the dynamically older clusters (with larger $\tau / t_{\mathrm{r} 1}$ values) tend to have shallower PDMFs. Although this result is based in only 5 clusters, we interpret this observed flattening trend as a dynamical effect, as also suggested by previous works for other systems (Kroupa et al. 2001; Baumgardt \& Makino 2003; Bonatto \& Bica 2005). In fact, it is hard not to expect some dynamical signature in the PDMFs for those LMC clusters with quite different dynamical ages. On the other hand, the PDMF flattening (that can reach $\Delta \alpha \sim 1.0$ ) could be interpreted as primordial mass segregation, at least in the case of the central regions. Or perhaps dynamical mass segregation in the LMC cluster's cores occurs fast enough that any age dependence of the PDMF would be seen only in a sample of young clusters. Again we stress the importance of $\mathrm{N}$-body simulations that configure a unique tool for providing reliable answers.

Although a scatter of $\sim 1.0$ in $\alpha$ is expected due to Poisson noise and the dynamical evolution of stellar clusters (Kroupa 2001), the aforementioned trends are in accordance with what one expects from dynamical arguments. In order to improve this dynamical investigation, and therefore confirm or reject these trends, we are applying our PDMF determination techniques to a larger number of CMDs of LMC/SMC clusters (Brocato et al. 2001).

Acknowledgements. We thank Sandro Javiel and Charles Bonatto for useful discussions. We acknowledge CNPq and PRONEX/FINEP 76.97.1003.00 for partially supporting this work. LOK acknowledges FAPESP postdoctoral fellowship 05/01351-5.

\section{References}

Andreuzzi, G., Buonanno, R., Fusi Pecci, F., Iannicola, G., \& Marconi, G. 2000, A\&A, 353, 944

Baumgardt, H., \& Makino, J. 2003, MNRAS, 340, 227

Beaulieu, S., Elson, R., Gilmore, G., et al. 1999, New Views of the Magellanic Clouds, IAU Symp. 190, ed. Y.-H. Chu, N. Suntzeff, J. Hesser, \& D. Bohlender, 460

Behrend, R., \& Meader, A. 2001, A\&A, 373, 190

Binney, J., \& Tremaine, S. 1987, Galactic Dynamics (Princeton University Press)

Bonatto, C., \& Bica, E. 2003, A\&A, 405, 525

Bonatto, C., \& Bica, E. 2005, A\&A, 437, 483

Bonnel, I. A., \& Davies, M. R. 1998, MNRAS, 295, 691

Brocato, E., Di Carlo, E., \& Menna, G. 2001, A\&A, 374, 523

Castro, R., Santiago, B., Gilmore, G., Beaulieu, S., \& Johnson, R. 2001, MNRAS, 326, 333 de Grijs, R., Gilmore, G., Johnson, R., \& Mackey, A. 2002a, MNRAS, 331, 245

de Grijs, R., Gilmore, G., Mackey, A., et al. 2002b, MNRAS, 337, 597

de Marchi, G., \& Paresce, F. 1996, ApJ, 467, 658

Durgapal, A. K., \& Pandey, A. K. 2001, A\&A, 375, 840

Girardi, L., Bressan, A., Bertelli, G., \& Chiosi, C. 2000, A\&AS, 141, 371

Gouliermis, D., Keller, S. C., Kontizas, M., Kontizas, E., \& Bellas-Velidis, I. 2004, A\&A, 416,137

Hillenbrand, L. A., \& Hartmann, L. W. 1998, ApJ, 492, 540

Howell, J. H., Guhathakurta, P., \& Tan, A. 2000, AJ, 119, 1259

Hurley, J., \& Tout, C. 1998, MNRAS, 300, 977

Kerber, L., \& Santiago, B. 2005, A\&A, 435, 77

Kerber, L., Santiago, B., Castro, R., \& Valls-Gabaud, D. 2002, A\&A, 390, 121

Kroupa, P. 2001, MNRAS, 322, 231

Kroupa, P. 2002, Science, 295, 88

Kroupa, P., Tout, C., \& Gilmore, G. 1991, MNRAS, 251, 293

Kroupa, P., Aarseth, S., \& Hurley, J. 2001, MNRAS, 321, 699

Mackey, A., \& Gilmore, G. 2003, MNRAS, 338, 85

Moraux, E., Kroupa, P., \& Bouvier, J. 2004, A\&A, 426, 75

Raboud, D., \& Mermilliod, J.-C. 1998, A\&A, 333, 897

Sagar, R., \& Richtler, T. 1991, A\&A, 250, 324

Salpeter, E. 1955, ApJ, 121, 161

Sandhu, T. S., Pandey, A. K., \& Sagar, R. 2003, A\&A, 408, 515

Santiago, B., Beaulieu, S., Johnson, R., \& Gilmore, G. 2001, A\&A, 369, 74

Spitzer, L. 1987, Dynamical Evolution of Globular Clusters (Princeton University Press) Stolte, A., Grebel, E., Brandner, W., \& Figer, D. F. 2002, A\&A, 394, 459

Tout, C. 1991, MNRAS, 250, 701 